\title{
Recent studies on electrical and magnetic properties of molecular solids
}

\author{
YUSEI MARUYAMA \\ Department of Materials Chemistry, College of Engineering, Hosei University, Kajinocho, \\ Koganei-shi, Tokyo 184, Japan

\begin{abstract}
Abtract. Recent progress in studies on electrical and magnetic properties of some typical molecular solids is described, focussing on research in Japan. Especially we focus on two recent topics, organic semiconductor BTQBT and organic superconductors. Two types of compound are discussed, (BEDT-TTF $)_{2} \mathrm{Cu}(\mathrm{NCS})_{2}$ and $\mathrm{M}_{3} \mathrm{C}_{60}(\mathrm{M}=\mathrm{K}$ or $\mathrm{Rb})$. Finally, two kinds of molecular ferromagnet, nitronyl nitroxide radicals and $\mathrm{C}_{60}$ (TDAE), are briefiy introduced.
\end{abstract}

Keywords. Molecular solids; electrical conductivity; Molecular fertomagnetism; organic superconductors; fullerenes.

\section{Electrical properties of organic molecular solids}

\subsection{Organic semiconductors and conductors}

The first observation of electrical conduction in organic solids was most confidently presented as a concept of "organic semiconductors" by Akamatu and Inokuchi (1950). Typical organic semiconductors at that time were such polycyclic aromatic hydrocarbons as anthracene, perylene or violanthrone. The essentially important feature in these substances is that they consist of $\pi$-electron systems, and it has been known that a variety of functionalities of organic materials are caused by these $\pi$-electron systems.

A breakthrough from semiconductors to organic metallic conductors was also opened by Akamatu et al (1954) as the discovery of very high conductivity of perylene-bromine complexes. The first observation of metallic conductivity was reported by Coleman et al (1973) for tetrathiafulvalene-tetracyano-quinodimethane (TTFTCNQ). The one-dimensional structure causes inherent instability in the electronic band structure and makes an energy gap at the Fermi-energy level. This electronic phase transition is accompanied by a lattice distortion through an electron-phonon coupling which stabilizes a charge-density-wave (CDW) insulating state. This type of metalinsulator transition usually occurs at a finite temperature, e.g. $53 \mathrm{~K}$ for TTF-TCNQ.

In order to stabilize metallic phases at low temperatures, the increase of dimensionality has been pursued by introducing intercolumnar interactions or heteroatomheteroatom interactions in plane-parallel directions between TTF-type molecules. Replacement of sulphur atoms in TTF moiety with selenium atoms which have larger interaction radius and smaller ionization potential is effective in increasing dimensionality. High-pressure application is physically effective in increasing dimensionality and in suppressing the metal-insulator transition.

Electron-electron repulsive correlation is another crucial factor in realization of a metallic state in narrow electron energy band systems. Especially, the on-site (on-one-molecule) Coulomb repulsive energy $(U)$ relative to the bandwidth $(W)$ is a very important measure in determining if the material will be metallic or insulating. If the ratio $U / W$ is much greater than unity, the material is a Mott-type insulator. In 
order to reduce $U$, various strategies have been attempted, such as to use larger-size, twin-type or multi-valency molecules. The former approach was very successful, that is, to use (bis)ethylenedithio-tetrathiafulvalene(BEDT-TTF) as an electron donor (Saito et al 1982).

1.1a $B T Q B T$ : Relatively highly conductive single-component organic semiconductors were recently realized by Yamashita et al (1991). A typical example is bis $[1,2,5]$ thiadiazolo-p-quinobis(1,3-dithiole) (BTQBT), which was synthesized with the strategy to realize much stronger intermolecular interactions in single-component molecular crystals. This novel molecule has electron-withdrawing heterocycles which are fused to the skeleton of a very strong electron donor, 2,2'-p-quinobis(1,3-dithiole) (QBT) (figure 1). In addition to the advantage of the strong intermolecular interactions (heteroatom-heteroatom contacts), relatively extending $\pi$-conjugation on the molecular plane of BTQBT may reduce the on-site Coulomb repulsion. Furthermore, this molecule is highly polarized because of intramolecular charge transfer, and accordingly it is expected to have a relatively small HOMO-LUMO gap. All these features may contribute to high electrical conductivity of this material.

BTQBT single crystals actually exhibited an unusually high conductivity of $1 \times 10^{-3} \mathrm{Scm}^{-1}$ at room temperature with a thermal activation energy of $0.21 \mathrm{eV}$. Hall effect and its temperature dependence were successfully observed by Imaeda et al (1992). The Hall mobility with positive sign is $2.4 \mathrm{~cm}^{2} \mathrm{~V}^{-1} \mathrm{~s}^{-1}$ at $330 \mathrm{~K}$ and $6.3 \mathrm{~cm}^{2} \mathrm{~V}^{-1} \mathrm{~s}^{-1}$ at $175 \mathrm{~K}$, and it changes with temperature $T^{-1 \cdot 6}$.

1.1b (DMe-DCNQI ${ }_{2} \mathrm{Cu}$ complex: The molecular structure of this compound is shown in figure 2. DMe-DCNQI is an electron-accepting molecule. The coordination to $\mathrm{Cu}$ ion is at the nitrogen atoms of cyanide groups. The DMe-DCNQI molecular planes overlap each other to form one-dimensional column structure in the crystal and copper ions connect these one-dimensional columns to form three-dimensional networks as shown in figure 3 . The copper ions are in mixed valence state of $4 / 3+$ average valence charge, which indicates that the copper $d$-orbitals are interacting with the $\pi$-orbitals (LUMO) of DMe-DCNQI molecules.

- The three-dimensional electronic structure stabilizes the metallic state down to low temperatures without metal-insulator transition. However, the metallic state is transformed to insulating state under relatively low pressure ( $>100 \mathrm{bar}$ ), and around the critical pressure region the "metal-insulator-metal" reentrant behaviour was observed with decreasing temperature (Tomic et al 1988). Similar metal-insulator transition

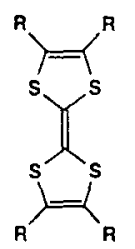

(a) $\mathrm{R}=\mathrm{H}$

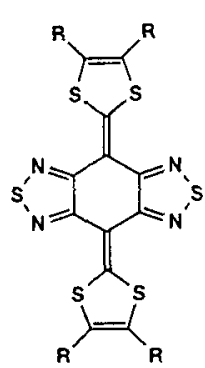

(b) $\mathrm{R}=\mathrm{H}$

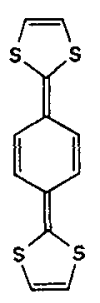

(c)

Figure 1. Molecular structures of some derivatives of $\operatorname{TTF}(\mathbf{a})$, BTQBT (b), and QBT (c). 


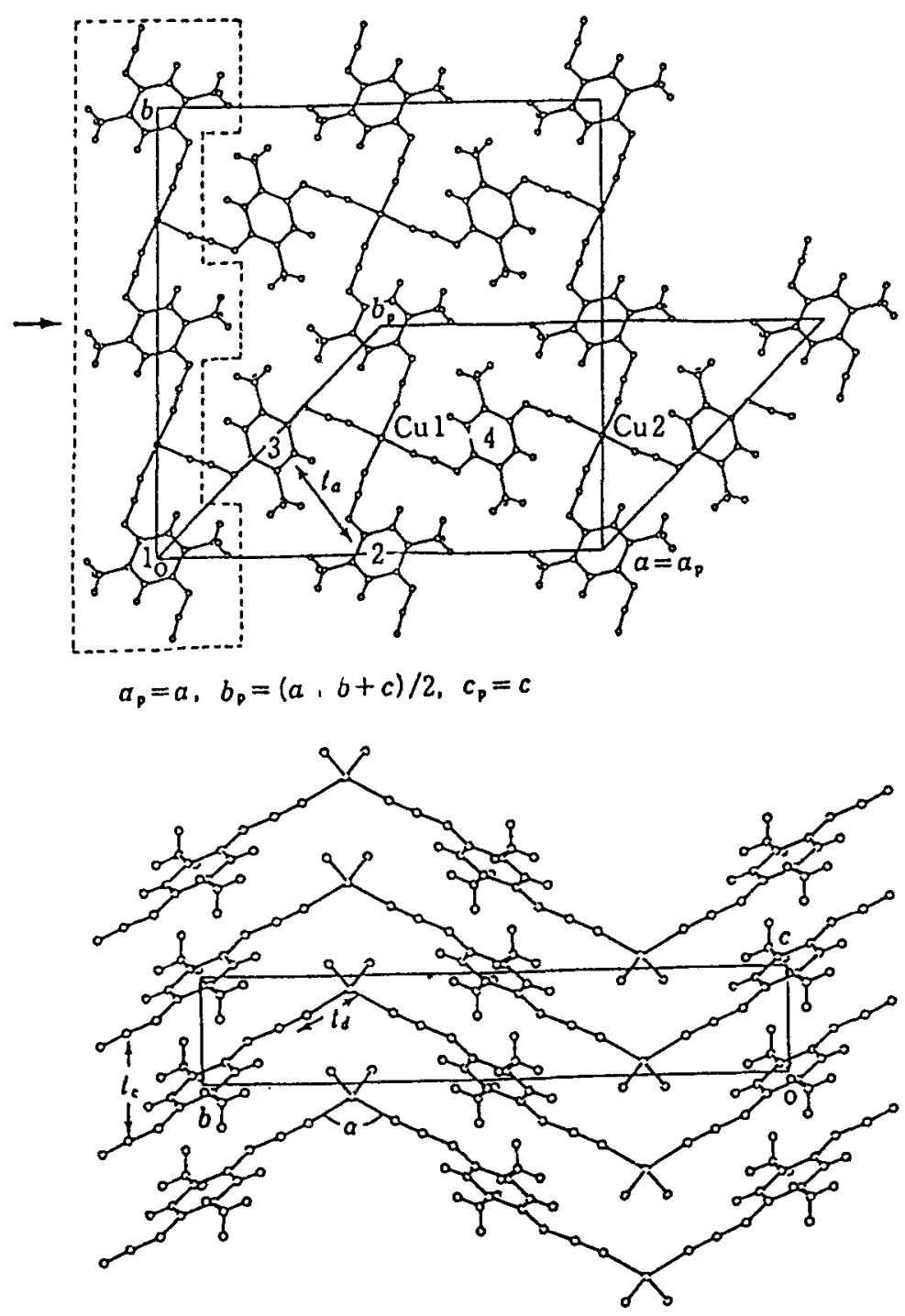

Figure 2. Crystal structure of (DMe-DCNQI $)_{2} \mathrm{Cu}$.

and also reentrant behaviour were discovered with the substitution of hydrogen atoms in DMe-DCNQI with deuteriums or ${ }^{12} \mathrm{C}$ of cyanide groups with ${ }^{13} \mathrm{C}$ (figure 4). The isotope exchange may cause qualitatively similar effect to the pressure effect, i.e. chemical pressure effect.

The theoretical details of the strongly correlated electron systems of (DMeDCNQI) ${ }_{2} \mathrm{Cu}$ crystal have been studied by Fukuyama (1993).

\subsection{Organic superconductors}

In order to stabilize metallic states at low temperatures, enhancement of low dimen- 


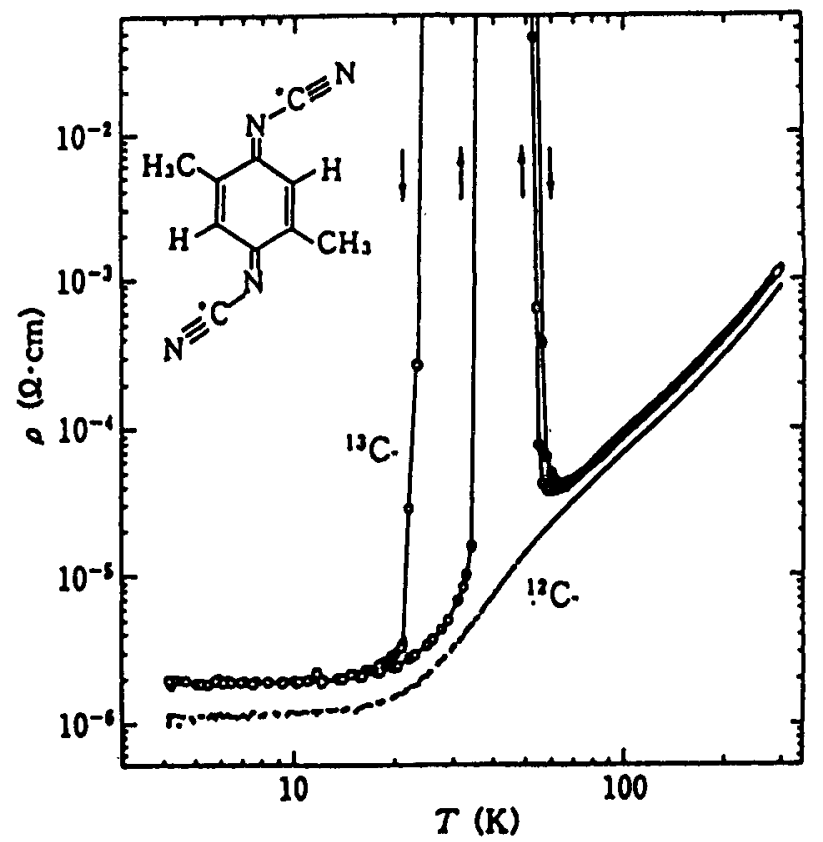

Figure 3. Reentrant metal-insulator transition by isotope substitution in (DMeDCNQI $)_{2} \mathrm{Cu}$.<smiles>[R2]C1=C([Y4])C(=NC#N)C([Y4])=C([R2])C1=NC#N</smiles>

\section{$R_{1}, R_{2} \cdot D C N Q I$}

Figure 4. Molecular structure of $R_{1}, R_{2}$-DCNQI.

sionality and reduction of on-site Coulomb repulsion energies have been pursued. The first organic superconductor discovered by Jerome et al (1980) is (TMTSF) 2 $\left(\mathrm{X}=\mathrm{PF}_{6}, T_{\mathrm{c}}=0.9 \mathrm{~K}\right.$ at $12 \mathrm{kbar}$ ) (figure 5). TMTSF complex forms quasi-one-dimensional columnar structure and the intercolumnar Se-Se interaction is essential to stabilize the metallic state. Although in the case of $\mathrm{X}=\mathrm{PF}_{6}$ compound some pressure is necessary to suppress the metal-insulator transition, $\mathrm{X}=\mathrm{ClO}_{4}$ compound was found to be superconducting at $1.4 \mathrm{~K}$ under ambient pressure due to the stronger intercolumnar interactions in this case. 
1.2a (BEDT-TTF $)_{2} X:$ (BEDT-TTF $)_{2} \mathrm{X}$ compounds (figure 5) investigated by Saito et al (1982) have given much more successful results. The larger size of $\pi$-electron conjugation of BEDT-TTF may reduce the on-site Coulomb repulsion and the twodimensional (not quasi-one-dimensional) crystal structure (figure 6) is advantageous for metallic phase.

The structure and function of the insulating layer $\mathrm{X}$ in (BEDT-TTF) ${ }_{2} \mathrm{X}$ are also important for two-dimensional metallic character of these compounds. In the cases of $\mathrm{X}=\mathrm{Cu}(\mathrm{NCS})_{2}, \mathrm{Cu}(\mathrm{CN})\left[\mathrm{N}(\mathrm{CN})_{2}\right], \mathrm{Cu}\left[\mathrm{N}(\mathrm{CN})_{2}\right] \mathrm{Br}$ and $\mathrm{Cu}\left[\mathrm{N}(\mathrm{CN})_{2}\right] \mathrm{Cl}$ the superconducting critical temperatures $T_{\mathrm{c}}$ are relatively high, 10.4, 12.3, 11.2 and $12.5 \mathrm{~K}$, respectively. The highest $T_{\mathrm{c}}$ is $13.1 \mathrm{~K}$ under $0.3 \mathrm{kbar}$ for $\mathrm{X}=\mathrm{Cu}\left[\mathrm{N}(\mathrm{CN})_{2}\right] \mathrm{Br}$. The pressure dependence of $T_{\mathrm{c}}$ for $\mathrm{X}=\mathrm{Cu}(\mathrm{NCS})_{2}$ is, however, rather negative. The isotope

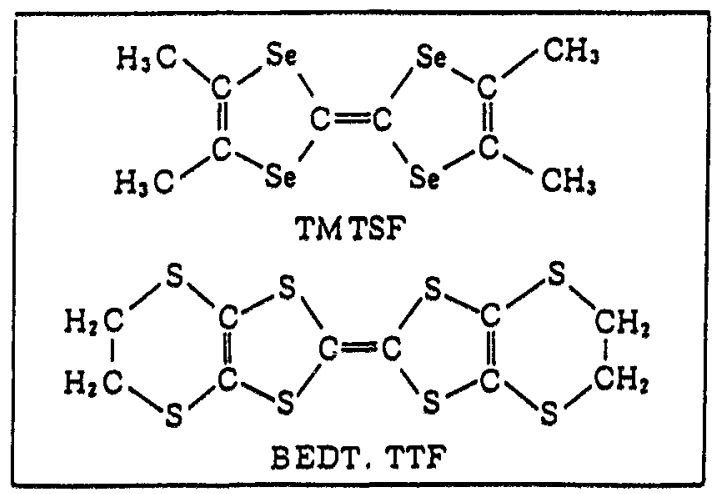

Figure 5. Molecular structures of the components of typical organic superconductors.
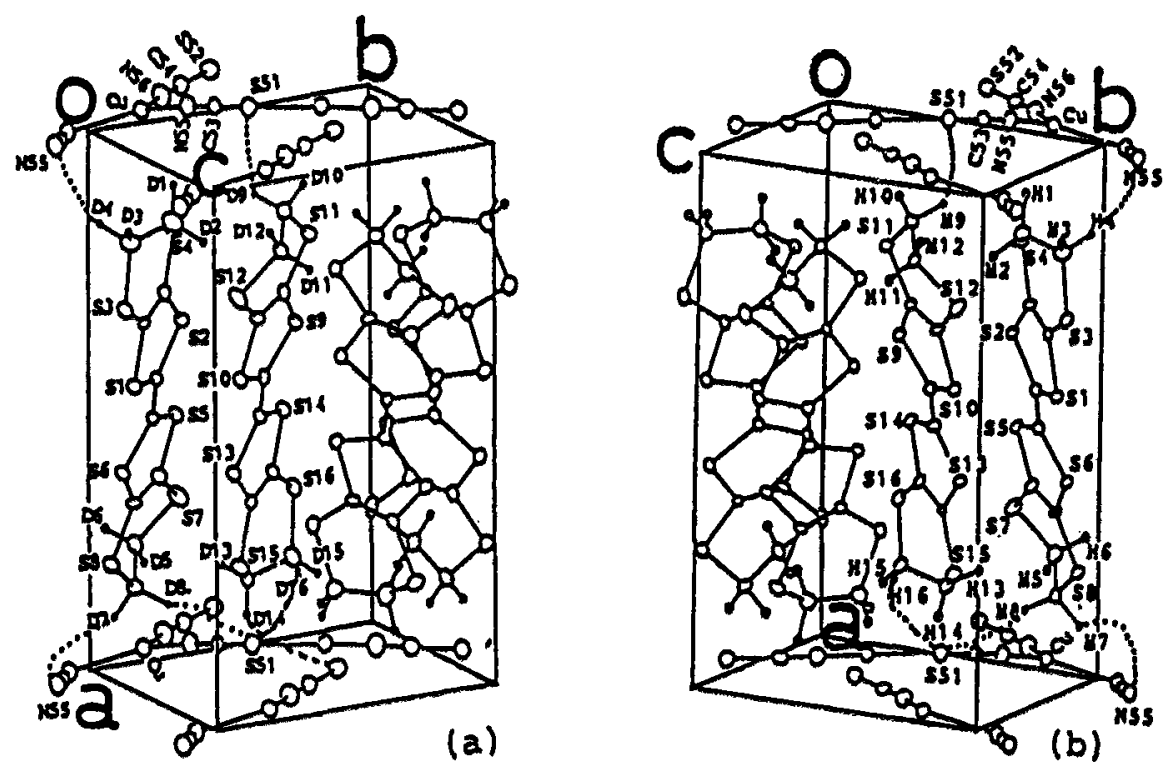

Figure 6. Crystal structure of (BEDT-TTF $)_{2} \mathrm{Cu}(\mathrm{NCS})_{2}$. 
effect for $T_{\mathrm{c}}$ is also negative for (BEDT-TTF $)_{2} \mathrm{Cu}(\mathrm{NCS})_{2}$ when the peripheral hydrogen atoms are substituted by deuterium atoms. On the contrary, substitution of ${ }^{12} \mathrm{C}$ atoms in inner TTF core with ${ }^{13} \mathrm{C}$ exhibited almost no isotope shift in $T_{\mathrm{c}}$. The magnetic field effects for metallic states have been very extensively studied to reveal the two-dimensional nature and Fermi-surface structures of these materials. (BEDTTTF $)_{2} \mathrm{X}$ compounds may be classified to type II superconductors, and the magnetic behaviours below $T_{\mathrm{c}}$ are not sufficiently clarified yet.

1.2b Fullerenes: The discovery of such new cage-type carbon-atom clusters as $\mathrm{C}_{60}$ or fullerenes has opened a new horizon in the field of molecular materials (Kroto et al 1985). After the development of mass-production method for fullerenes by carbon contact arc discharge (Kratschmer et al 1990), the discovery of superconductivity at $18 \mathrm{~K}$ in $\mathrm{K}_{3} \mathrm{C}_{60}$ (Hebard et al 1991) has strongly accelerated the study of fullerenes.

The electrical conductivity of $\mathrm{C}_{60}$ single crystal is strongly and negatively dependent on the ambient oxygen pressure, and it is believed to be around $10^{-5} \mathrm{~S} \mathrm{~cm}^{-1}$ at $295 \mathrm{~K}$ in the most oxygen-free pure $\mathrm{C}_{60}$ single crystals. The thermal activation energy of conductivity is $0.26 \mathrm{eV}$, which indicates that pure $\mathrm{C}_{60}$ crystal is a molecular semiconductor. The charge-carrier mobility in pure $\mathrm{C}_{60}$ single crystals was measured by two methods, time-of-flight (Frankevich et al 1993) and Hall effect (Arai 1994). The drift mobilities obtained by the former method were $1.7 \mathrm{~cm}^{2} \mathrm{~V}^{-1} \mathrm{~s}^{-1}$ for holes and $0.5 \mathrm{~cm}^{2} \mathrm{~V}^{-1} \mathrm{~s}^{-1}$ for electrons which are temperature-independent below $(50-250 \mathrm{~K})$ and above $(260-300 \mathrm{~K})$ the phase transition point, around $260 \mathrm{~K}$. At this temperature the mobility increased stepwise by 1.7 times with decreasing temperature. It is known that the changes in the molecular rotational freedom and accordingly in the crystal structure take place at this temperature, which suggests that transporting electrons or holes may couple with molecular rotations. The Hall mobility with positive sign is reported to be $0.15 \mathrm{~cm}^{2} \mathrm{~V}^{-1} \mathrm{~s}^{-1}$ and it is again almost temperature-independent between $290 \mathrm{~K}$ and $570 \mathrm{~K}$.

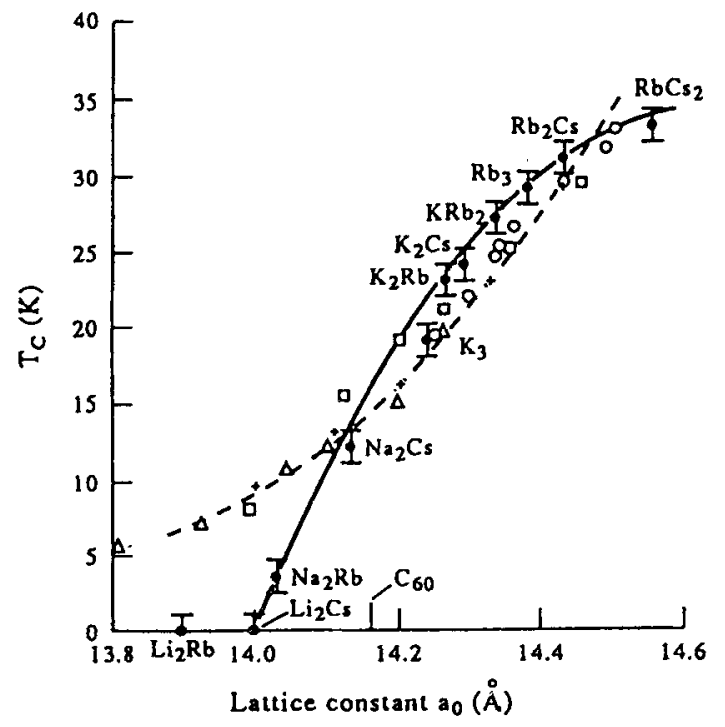

Figure 7. Correlation between $T_{\mathrm{c}}$ and $a_{0}$ in alkali metal- $\mathrm{C}_{60}$ superconductors. 
$\mathrm{C}_{60}$ crystals doped with alkali metals or alkaline-earth metals, $\mathbf{M}_{x} \mathrm{C}_{60}$, have realized a new family of molecular metals and superconductors which have much higher $T_{\mathrm{c}}$ than organic superconductors known so far. The dominant stoichiometry which yields metallic state is $x=3$ and the corresponding ones for alkali metal-doped complexes, fullerides, make a systematic family of molecular superconductors (figure 7). The highest $T_{\mathrm{c}}$ in this family is $33 \mathrm{~K}$ for $\mathrm{Cs}_{2} \mathrm{RbC}_{60}$. However, it was very recently reported that $\mathrm{Cs}_{3} \mathrm{C}_{60}$ stabilized under pressure may have $\sim 40 \mathrm{~K}$ for $T_{\mathrm{c}}$. The intrinsic nature of the metallic state and the mechanism of superconductivity in fullerides are not yet clarified. In the BCS regime a probable theoretical approach based on intramolecular-vibration-mediated Cooper pairing was proposed taking into account a strongly correlated electron system and relevant phonons and Coulomb energy windows (Ivanov and Maruyama 1995). However, such different approaches as charge-transfer pairing could be more general and relevant to molecular superconductors.

\section{Magnetic properties of organic molecular solids}

A very important subject in this field is to create or synthesize molecular ferromagnets. We focus on this subject in this section.

\subsection{Molecular ferromagnetism}

As radical molecules - neutral or charged - have unpaired electrons and show paramagnetism, they could be candidates for ferromagnetic materials. However, once these paramagnetic species are in the solid state, as a rule they are Curie-Weiss paramagnets. If the interactions between spins in different molecules are strong enough to order at some temperatures, the anti-ferromagnetically ordered states usually overcome the ferromagnetic states, since the bonding states between molecules are usually more stable than the antibonding states. Therefore the next strategy is how to make the bonding states unstable, or to make two unpaired spin states orthogonal.

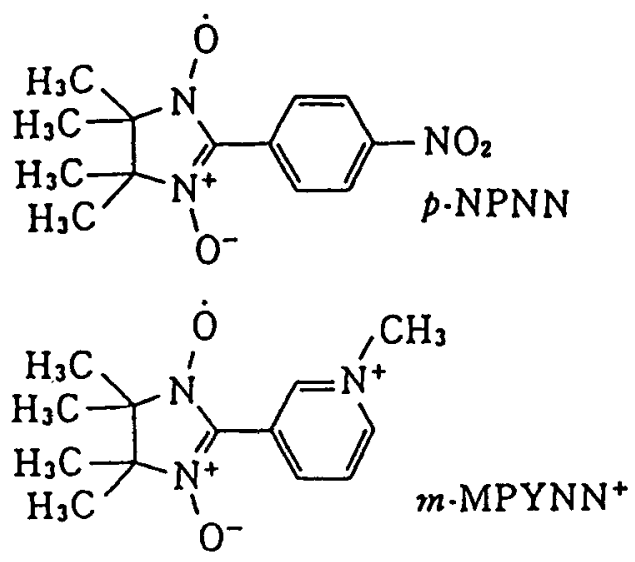

Figure 8. Molecular structures of nitronyl nitroxide radicals. 

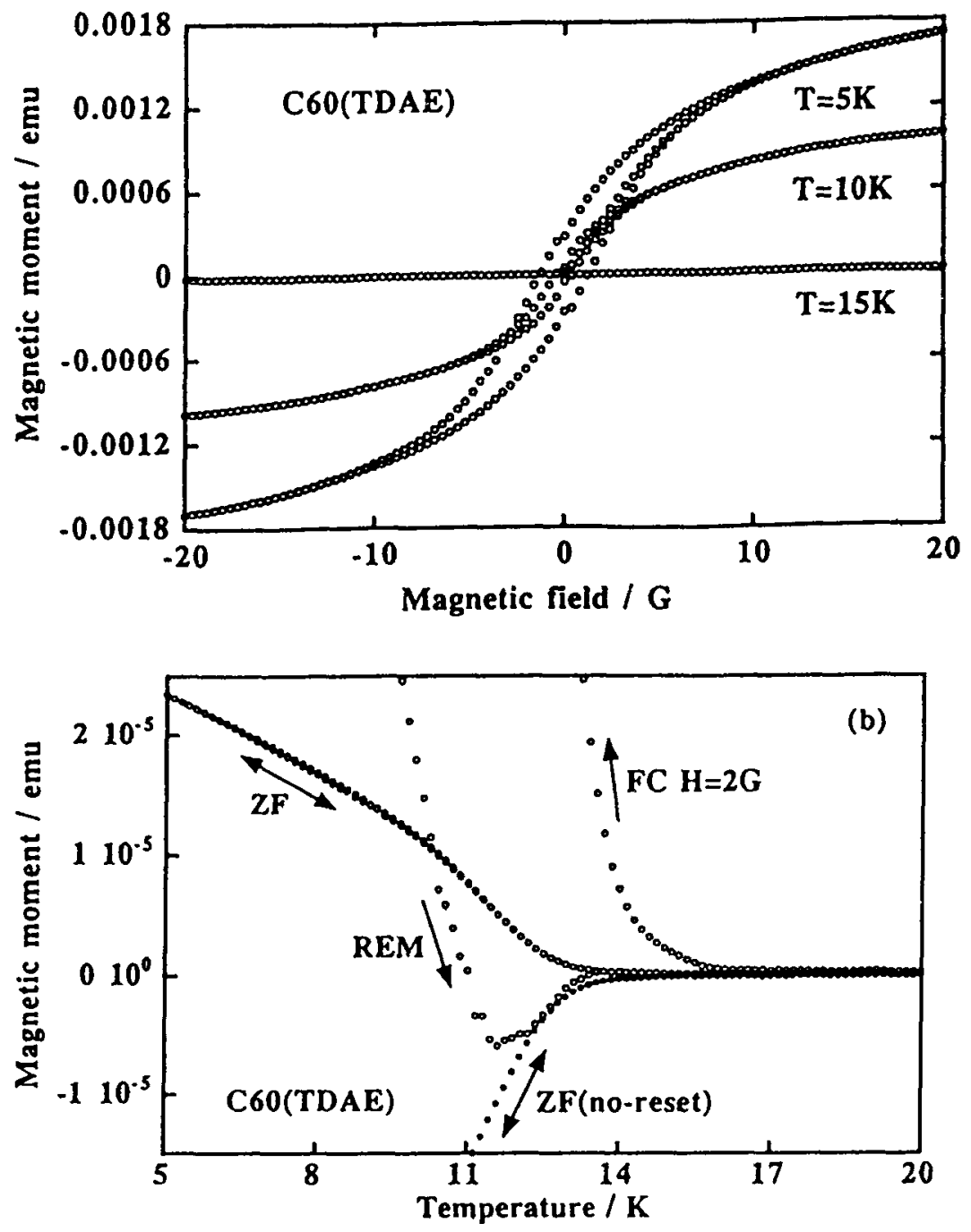

Figure 9. Spontaneous magnetization in $\mathrm{C}_{60}$ (TDAE).

2.1a Nitronyl nitroxide radicals: Nitronyl nitroxide radicals are the successful examples in this line (figure 8) (Kinoshita et al 1991). In the crystal unpaired spin states on -NO groups are rather localized and located very far from each other due to crystal stability. Thus, nearly orthogonal relation between two unpaired spin states in neighbouring molecules is fulfilled in this case. Further, large on-site Coulomb repulsion may contribute to stabilize the triplet or ferromagnetic states in this system. This compound shows ferromagnetic ordering below $0.65 \mathrm{~K}$.

2.1b $C_{60}(T D A E)$ ferromagnet: $C_{60}(T D A E)$ complex, where TDAE means tetrakisdiamino-ethylene, could be a different approach for a molecular ferromagnet in the charge transfer mechanism (Allemand et al 1991). Although extensive studies of this system have revealed aspects of its magnetic behaviour, such as the spontaneous 
magnetization below $13 \mathrm{~K}$ or the hysteresis curve in the isothermal magnetization (figure 9) (Suzuki et al 1994), the nature of the magnetism, whether really ferromagnetic or spin-glass-like, is still unclear. The quest for iteneracy of ferromagnetic spins is almost known to be negative through the recent experiments by two groups (Maruyama and Blinc).

\section{References}

Akamatu $\mathrm{H}$ and Inokuchi $\mathrm{H} 1950 \mathrm{~J}$. Chem. Phys. 18810

Akamatu H, Inokuchi $\mathrm{H}$ and Matsunaga Y 1954 Nature 173168

Allemand P M, Khemani K C. Koch A, Wudl F and Thompson J D 1991 Science 253301

Arai T 1994 Electrical properties of $C_{60}$ single crystals, Doctor Thesis, University of Tokyo, Tokyo

Coleman L B, Cohen M N, Sandman D J, Yamagishi F F, Garito A F and Heeger A J 1973 Solid State Commun. 121125

Frankevich E, Maruyama Y and Ogata H 1993 Chem. Phys. Lett. 21439

Fukuyama H 1993 J. Phys. Soc. Jpn 621436

Hebard A F et al 1991 Nature 350600

Imaeda K, Yamashita Y, Li Y, Mori T. Inokuchi H and Sano M 1992 J. Mater. Chem. 2115

Ivanov V and Maruyama Y 1995 Physica $C$ (in press)

Jerome D, Mazaud A, Ribault Mí and Bechgaard K 1980 J. Phys. Lett. 41 L95

Kinoshita M et al 1991 Chem. Lett. 1225

Kratschmer W, Lamb L D, Fostiropoulos K and Huffman D R 1990 Nature 347354

Kroto H W, Heath J R, O'Brien S C, Curl R F and Smalley R E 1985 Nature 318162

Maruyama $Y$ and Blinc R (Two groups)

Saito G. Enoki T and Inokuchi H 1982 Chem. Lett. 1345

Suzuki A, Suzuki T, Whitehead R J and Maruyama Y 1994 Chem. Phys. Lett. 223517

Tomic S, Jerome D, Aumuller A, Erk P, Hunig S and von Schulz J U 1988 Synth. Met. 27 B281

Wudl F and Thompson J D 1992 J. Phys. Chem. Solids 531499

Yamashita Y, Tanaka S, Imaeda K and Inokuchi H 1991 Chem. Lett. 1213 